\title{
Conversion of viridicatic acid to crustosic acid by cytochrome P450 enzyme-catalysed hydroxylation and spontaneous cyclisation
}

\author{
Jenny Zhou ${ }^{1}$. Shu-Ming Li ${ }^{1}$ (i)
}

Received: 10 September 2021 / Revised: 26 October 2021 / Accepted: 28 October 2021 / Published online: 11 November 2021

(c) The Author(s) 2021

\begin{abstract}
Cytochrome $\mathrm{P} 450$ monooxygenases (P450s) are considered nature's most versatile catalysts and play a crucial role in regioand stereoselective oxidation reactions on a broad range of organic molecules. The oxyfunctionalisation of unactivated carbon-hydrogen $(\mathrm{C}-\mathrm{H})$ bonds, in particular, represents a key step in the biosynthesis of many natural products as it provides substrates with increased reactivity for tailoring reactions. In this study, we investigated the function of the P450 enzyme $\mathrm{TraB}$ in the terrestric acid biosynthetic pathway. We firstly deleted the gene coding for the DNA repair subunit protein Ku70 by using split marker-based deletion plasmids for convenient recycling of the selection marker to improve gene targeting in Penicillium crustosum. Hereby, we reduced ectopic DNA integration and facilitated genetic manipulation in P. crustosum. Afterward, gene deletion in the $\Delta k u 70$ mutant of the native producer P. crustosum and heterologous expression in Aspergillus nidulans with precursor feeding proved the involvement of $\mathrm{TraB}$ in the formation of crustosic acid by catalysing the essential hydroxylation reaction of viridicatic acid.
\end{abstract}

\section{Key points}

-Deletion of Ku70 by using split marker approach for selection marker recycling.

- Functional identification of the cytochrome P450 enzyme TraB.

- Fulfilling the reaction steps in the terrestric acid biosynthesis.

Keywords Cytochrome P450 · Hydroxylation · Terrestric acid biosynthesis $\cdot$ Split marker approach · Marker recycling

\section{Introduction}

Natural products (NPs) isolated from microorganisms are well-known for their biological activities and are promising candidates for drug development (Katz and Baltz 2016). Fungi, especially those isolated from the marine environment, are reported to produce many NPs that exhibit antibacterial, anti-fungal, anti-oxidant, anti-inflammatory, and even cytotoxic activities and have therefore largely contributed to the expansion of the pharmaceutical repertoire (Keller 2019; Shabana et al. 2021). Recent advances in targeted gene manipulation, including gene disruption and heterologous expression, allowed the analysis of gene functions and

Shu-Ming Li

shuming.li@staff.uni-marburg.de

1 Institut Für Pharmazeutische Biologie Und Biotechnologie, Fachbereich Pharmazie, Philipps-Universität Marburg, Robert-Koch-Straße 4, 35037 Marburg, Germany the elucidation of NP biosynthetic pathways (Atanasov et al. 2021).

Cytochrome P450 enzymes (P450s) are a ubiquitously occurring superfamily of heme-thiolate proteins and are distributed in all kingdoms of life, including humans, animals, plants, fungi, and bacteria (Harken and Li 2021; McIntosh et al. 2014; Zhang et al. 2021). They catalyse oxidation reactions in the biosynthesis of many natural products, such as alkaloids, terpenes, and polyketides. These reactions are involved in the functionalisation of non-reactive carbons by aliphatic and aromatic bond hydroxylation, epoxidation, heterocyclisation, ring-coupling, and $\mathrm{C}-\mathrm{C}$ bond cleavage and are often performed with remarkable regio- and stereoselectivity (Podust and Sherman 2012).

Our group recently identified a tetronic acid biosynthetic gene cluster (pcr11009-pcr11016, traA-H) in Penicillium crustosum PRB-2 (Fan et al. 2019, 2020). Biochemical investigation proved the function of the hybrid PKS-NRPS TraA and the enoyl reductase TraG for the formation of 
viridicatic acid (1), which is subsequently hydroxylated and undergoes ring formation to yield crustosic acid (2). The nonheme $\mathrm{Fe}^{\mathrm{II}}$-2OG-dependent oxygenase TraH catalyses an oxidative decarboxylation of $\mathbf{2}$, followed by stereospecific reduction with the flavin-containing oxidoreductase TraD to form the final product terrestric acid (3) (Fan et al. 2019, 2020) (Fig. 1). TraB from this cluster was proposed as the responsible enzyme for the hydroxylation reaction of $\mathbf{1}$. To verify TraB function, Fan et al. performed gene deletion using the $h p h$ gene as selective marker (Fan et al. 2019). However, deletion of traB in P. crustosum did not lead to the accumulation of $\mathbf{1}$. Instead, accumulation of clavatol, hydroxyclavatol, and hydroxyclavatol methyl ether was detected, which resembled the secondary metabolite profile of the $\Delta t r a A$ deletion mutant reported in the same study. A possible reason for these unexpected results could be the unintentional polar effect on $\operatorname{tra} A$. In this study, we repeated the $t r a B$ deletion in P. crustosum by preparing a new deletion cassette and proved its function as the proposed hydroxylase.

\section{Materials and methods}

\section{Strains, media, and culture conditions}

Fungal strains used and created in this study are listed in Table S1 (Supplementary Information). Saccharomyces cerevisiae strain HOD114-2B was used for cloning via homologous recombination. The yeast was grown at $30^{\circ} \mathrm{C}$ in YPD medium (1\% yeast extract, $2 \%$ peptone, and $2 \%$ glucose). For selection of transformants, synthetic complete medium without uracil $(6.7 \mathrm{~g} / \mathrm{L}$ yeast nitrogen base with ammonium sulphate, $650 \mathrm{mg} / \mathrm{L}$ CSM-His-Leu-Ura, $20 \mathrm{mg} / \mathrm{L}$ histidine, and $60 \mathrm{mg} / \mathrm{L}$ leucine) was used.

Escherichia coli strain DH5 $\alpha$ was used for plasmid propagation and cultivated at $37{ }^{\circ} \mathrm{C}$ in LB medium (lysogeny broth). For selection of recombinant strains, ampicillin $(50 \mathrm{mg} / \mathrm{L})$ was added to the medium.

Penicillium crustosum strain PRB-2 (Wu et al. 2012; Yu et al. 2019) was deposited as strain MFCSCS 200 in the culture collection of the Key Laboratory of Marine Drugs, the Ministry of Education of China, School of Medicine and Pharmacy, Ocean University of China, Qingdao, People's Republic of China. All pyrG intact strains were cultivated standing at $25^{\circ} \mathrm{C}$ in $\mathrm{PDB}$ medium $(24 \mathrm{~g} / \mathrm{L}$ potato dextrose broth, Sigma-Aldrich). pyrG deficient strains were cultivated on PDB medium supplemented with $0.5 \mathrm{~g} / \mathrm{L}$ uridine and $0.5 \mathrm{~g} / \mathrm{L}$ uracil. For $p y r G$ marker recycling, $7.5 \mathrm{mM} 5$-fluoroorotic acid (5-FOA), $0.5 \mathrm{~g} / \mathrm{L}$ uridine, and $0.5 \mathrm{~g} / \mathrm{L}$ uracil were added to the medium at $\mathrm{pH} 4$.

Aspergillus nidulans strains were cultivated standing at $37{ }^{\circ} \mathrm{C}$ in PDB medium supplemented with $0.5 \mathrm{~g} / \mathrm{L}$ uridine, $0.5 \mathrm{~g} / \mathrm{L}$ uracil, $2.5 \mathrm{mg} / \mathrm{L}$ riboflavin, and/or $0.5 \mathrm{mg} / \mathrm{L}$ pyridoxine, respectively, depending on the used selection markers.

\section{Isolation of fungal genomic DNA}

For isolation of genomic DNA, P. crustosum and A. nidulans strains were cultivated in $500 \mu \mathrm{L}$ LMM medium $(1.0 \%$ glucose, $50 \mathrm{~mL} / \mathrm{L}$ salt solution, $1 \mathrm{~mL} / \mathrm{L}$ trace element solution, and $0.5 \%$ yeast extract) with appropriate supplementation for 3 days at $30^{\circ} \mathrm{C}$. The mycelia were then collected by centrifugation at $13,300 \mathrm{rpm}$ for $10 \mathrm{~min}$. Four hundred microlitres of LETS-buffer (10 mM Tris- $\mathrm{HCl} \mathrm{pH}$ 8.0, $20 \mathrm{mM}$ EDTA, 0,5\% SDS, $0.1 \mathrm{M} \mathrm{LiCl}$ ) were added, and the mycelia were crushed with glass beads in a MiniLys homogeniser (Bertin Technologies, Montigny-le-Bretonneux. France) for 3 min at full speed. After adding $300 \mu \mathrm{L}$ LETS-buffer, the solution was treated with $700 \mu \mathrm{L}$ phenol/ chloroform/isoamyl alcohol (25:24:1), mixed by inverting and centrifuged at 13,000 rpm for $10 \mathrm{~min}$. Subsequently, the aqueous phase was treated with $900 \mu \mathrm{L}$ absolute ethanol for DNA precipitation and centrifuged at 13,300 rpm, $4{ }^{\circ} \mathrm{C}$ for $30 \mathrm{~min}$. The DNA was washed with $500 \mu \mathrm{L} 70 \%$ ethanol, dried, and dissolved in $50 \mu \mathrm{L}$ distillated $\mathrm{H}_{2} \mathrm{O}$.

\section{Genome sequencing and sequence analysis}

Genome sequencing was performed by BerryGenomics (Beijing, China) using Novaseq6000/X-ten (Illumina). The prediction of open reading frames was performed using secondFind (http://biosyn.nih.go.jp/2ndfind/). Enzyme functions were predicted with online BLAST approaches (http://blast.ncbi.nlm.nih.gov). The nucleotide sequence of the terrestric acid cluster reported in this study is available

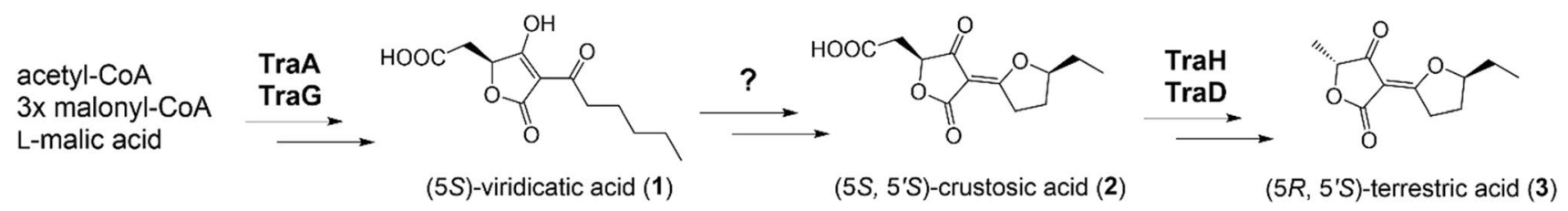

Fig. 1 Proposed biosynthetic pathway of terrestric acid in P. crustosum PRB-2 modified after Fan et al. (2020) 
at GenBank under accession number MK360919 and that of Ku70 under KAF7525404.1.

\section{PCR amplification, gene cloning, and plasmid construction}

Plasmids generated and used in this study are listed in Table S2. The primers used for PCR amplification were synthesised by Seqlab GmbH (Göttingen, Germany) and are listed in Table S3. For PCR amplification, Phusion HighFidelity DNA polymerase from New England Biolabs (NEB) and a T100TM Thermal cycler from Bio-Rad were used.

Plasmids for gene deletion and heterologous expression were constructed by homologous recombination in Escherichia coli or Saccharomyces cerevisiae. For gene deletion experiments followed by subsequent marker recycling, a deletion cassette was prepared using two split marker constructs. One of them contained the approximately $1.5 \mathrm{kbps}$ upstream DNA sequence of the target gene, $300 \mathrm{bps}$ from the downstream region for subsequent marker recycling, and two-thirds of the afpyr $G$ selection marker with 25-30 bps overlap to the SmaI restriction site of the pESC-URA vector. The second construct consists of another two-thirds of the afpyr $G$ cassette, the approximately $1.5 \mathrm{kbps}$ downstream DNA sequence of the target gene, and was also introduced into the SmaI restriction site of linearised vector pESCURA. The afpyrG resistance cassette originated from $\mathrm{pFK} 23$ (Kindinger et al. 2019).

The plasmid for heterologous expression of traB in $A$. nidulans was constructed by PCR amplification of $\operatorname{traB}$ including its terminator of $632 \mathrm{bp}$ from genomic DNA of $P$. crustosum and subsequent cloning into $S f o \mathrm{I}$-linearised vector pJN017 (Kindinger et al. 2019) containing the flanking regions of the $w A$ gene, the $g p d A$ promoter, and the afribo selection marker. The amplified genomic sequence of $t r a B$ was cloned between the gpdA promoter and the afribo marker by using 25-30 bps overlap to the $S f o$ I restriction site.

\section{Genetic manipulation in P. crustosum FK15, JZ02, and $A$. nidulans L08030}

Transformation of $P$. crustosum FK15 (Kindinger et al. 2019), JZ02, and A. nidulans LO8030 (Chiang et al. 2016) was performed by polyethylene glycol (PEG)-mediated protoplast transformation. Fresh spores of FK15 or JZ02, respectively, were inoculated in a $250-\mathrm{mL}$ flask containing $30 \mathrm{~mL}$ LMM medium (1.0\% glucose, $50 \mathrm{~mL} / \mathrm{L}$ salt solution, $1 \mathrm{~mL} / \mathrm{L}$ trace element solution, and $0.5 \%$ yeast extract) supplemented with $0.5 \mathrm{~g} / \mathrm{L}$ uridine and $0.5 \mathrm{~g} / \mathrm{L}$ uracil and incubated at $25{ }^{\circ} \mathrm{C}, 230 \mathrm{rpm}$ for $11 \mathrm{~h}$. Spores of LO8030 were inoculated in a $250-\mathrm{mL}$ flask containing $30 \mathrm{~mL} \mathrm{LMM}$ medium supplemented with $0.5 \mathrm{~g} / \mathrm{L}$ uridine, $0.5 \mathrm{~g} / \mathrm{L}$ uracil,
$2.5 \mathrm{mg} / \mathrm{L}$ riboflavin, and $0.5 \mathrm{mg} / \mathrm{L}$ pyridoxine and incubated at $37^{\circ} \mathrm{C}, 230 \mathrm{rpm}$ for $5 \mathrm{~h}$. The germlings were collected by centrifugation at $5000 \mathrm{rpm}$ for $5 \mathrm{~min}$ and washed with $30 \mathrm{~mL}$ distillated $\mathrm{H}_{2} \mathrm{O}$ and $10 \mathrm{~mL}$ osmotic buffer $(1.2 \mathrm{M}$ $\mathrm{MgSO}_{4}$ in $10 \mathrm{mM}$ sodium phosphate, $\mathrm{pH}$ 5.8). For cell wall degradation, the germlings were transferred into a $100-\mathrm{mL}$ flask containing $10 \mathrm{~mL}$ osmotic buffer, $50 \mathrm{mg}$ lysing enzyme from Trichoderma harzianum (Sigma), and $20 \mathrm{mg}$ yatalase from Corynebacterium sp. OZ-21 (OZEKI Co., Ltd.) and incubated at $30^{\circ} \mathrm{C}, 100 \mathrm{rpm}$. After $3 \mathrm{~h}$, the protoplasts were transferred into a $50-\mathrm{mL}$ falcon tube, overlaid with trapping buffer ( $0.6 \mathrm{M}$ sorbitol in $0.1 \mathrm{M}$ Tris-HCI, pH 7.0), and centrifuged at $5000 \mathrm{rpm}$ for $15 \mathrm{~min}$ at $4{ }^{\circ} \mathrm{C}$. The cells were then collected from the interphase and carefully transferred into a $15-\mathrm{mL}$ falcon tube. After centrifugation at $5000 \mathrm{rpm}$ for $7 \mathrm{~min}$, the pellet was resuspended in $200 \mu \mathrm{L}$ cold STC buffer (1.2 $\mathrm{M}$ sorbitol, $10 \mathrm{mM} \mathrm{CaCl}_{2}$, and $10 \mathrm{mM}$ Tris-HCI, $\mathrm{pH}$ 7.5). For transformation, the protoplasts were treated with $3 \mu \mathrm{g}$ DNA and incubated on ice for $50 \mathrm{~min}$. Then, $1.25 \mathrm{~mL}$ PEG solution (60\% PEG 4000, $50 \mathrm{mM} \mathrm{CaCl}_{2}, 50 \mathrm{mM}$ Tris$\mathrm{HCI}, \mathrm{pH}$ 7.5) was added, gently mixed, and incubated at room temperature for $25 \mathrm{~min}$. Five millilitres of STC buffer was added to the mixture, which was then transferred onto plates with SMM bottom medium $(1.0 \%$ glucose, $50 \mathrm{~mL} / \mathrm{L}$ salt solution, $1 \mathrm{~mL} / \mathrm{L}$ trace element solution, $1.2 \mathrm{M}$ sorbitol, $1.6 \%$ agar). The plates were overlaid with SMM top medium $(1.0 \%$ glucose, $50 \mathrm{~mL} / \mathrm{L}$ salt solution, $1 \mathrm{~mL} / \mathrm{L}$ trace element solution, $1.2 \mathrm{M}$ sorbitol, and $0.8 \%$ agar) and incubated at $25^{\circ} \mathrm{C}$ for 5 days for $P$. crustosum or $37^{\circ} \mathrm{C}$ for 3 days for $A$. nidulans. For A. nidulans LO8030, the SMM bottom and top media were additionally supplemented with $0.5 \mathrm{~g} / \mathrm{L}$ uridine, $0.5 \mathrm{~g} / \mathrm{L}$ uracil, and $0.5 \mathrm{mg} / \mathrm{L}$ pyridoxine. The transformants were transferred onto fresh GMM plates $(1.0 \%$ glucose, $50 \mathrm{~mL} / \mathrm{L}$ salt solution, $1 \mathrm{~mL} / \mathrm{L}$ trace element solution, and $1.6 \%$ agar) with appropriate supplementation and, subsequently, inoculated for the isolation of genomic DNA to verify positive transformants via PCR.

\section{PCR verification of positive transformants and cultivation for secondary metabolite production}

Genomic DNA was isolated from the transformants and used for PCR amplification. PCR for the control of gene deletion transformants was performed using one primer binding outside of the deletion cassette and the second one binding in the marker gene. To confirm deletion of the afpyrG gene by marker recycling, primers were designed to amplify the flanking regions with or without the $p y r G$ sequence to determine if the selection marker is present. For $t r a B$ overexpression strains, primers binding outside of the expression construct and the counterpart binding in the afribo marker or the gpdA promoter, respectively, were used. In addition, 
the presence of $t r a B$ was proven by PCR amplification of the expressed gene. The PCR results are given in Figs. S1, S2, S3, and S4.

For detection of secondary metabolites, transformants were cultivated in PDB medium with the required supplements. After 14 days, secondary metabolites were extracted with equivalent volumes of ethyl acetate for three times, dissolved in a mixture of methanol and distilled $\mathrm{H}_{2} \mathrm{O}(9: 1)$ and analysed via LC-MS.

\section{Precursor feeding in $A$. nidulans traB expression strain}

Five millilitres of PDB medium supplemented with $0.5 \mathrm{~g} / \mathrm{L}$ uridine, $0.5 \mathrm{~g} / \mathrm{L}$ uracil, and $0.5 \mathrm{mg} / \mathrm{L}$ pyridoxine was inoculated with spores of $A$. nidulans traB expression strain JZ01 or empty vector control strain BK06, respectively. Substrate 1 was dissolved in DMSO to give a $200 \mathrm{mM}$ stock solution and fed to the PDB cultures directly after inoculation in an appropriate amount to a final concentration of $0.2 \mathrm{mM}$. The cultures were incubated standing at $25^{\circ} \mathrm{C}$ for 7 days. Secondary metabolites were then extracted with equivalent volumes of ethyl acetate for three times, dissolved in a mixture of methanol and distilled $\mathrm{H}_{2} \mathrm{O}(9: 1)$, and analysed via LC-MS.

\section{Large-scale fermentation, extraction, and isolation of secondary metabolites}

To isolate $\mathbf{1}$, spores of the $P$. crustosum $\Delta$ traB mutant strain were inoculated into $20 \times 250 \mathrm{~mL}$ Erlenmeyer flasks containing $100 \mathrm{~mL}$ PDB liquid medium each and cultivated at $25^{\circ} \mathrm{C}$ under static conditions for 14 days. After separating mycelia by filtration, the supernatant was extracted with equal volumes of ethyl acetate for three times and evaporated under reduced pressure to give a crude extract $(1.2 \mathrm{~g})$. For purification, the crude extract was subjected to silica gel column chromatography and eluted with a gradient of $\mathrm{MeOH} / \mathrm{CH}_{2} \mathrm{Cl}_{2}$ (9:1 to $1: 1$ ) to give 16 fractions. Fraction 12-14 were pooled and evaporated under reduced pressure to obtain $1(293 \mathrm{mg})$.

To isolate 2 , spores of $A$. nidulans traB expression strain JZ01 were inoculated into two $250-\mathrm{mL}$ Erlenmeyer flasks containing $100 \mathrm{~mL}$ PDB liquid medium each, supplemented with $0.5 \mathrm{~g} / \mathrm{L}$ uracil, $0.5 \mathrm{~g} / \mathrm{L}$ uridine, and $0.5 \mathrm{mg} / \mathrm{L}$ pyridoxine. Thirty milligrammes of substrate $\mathbf{1}$ isolated from the P. crustosum $\triangle$ traB mutant strain was dissolved in DMSO, fed to the PDB cultures, and cultivated on a rotary shaker at $200 \mathrm{rpm}$ and $25^{\circ} \mathrm{C}$ for 8 days. After separating mycelia by filtration, the supernatant was extracted with equal volumes of ethyl acetate for three times and evaporated under reduced pressure to give a crude extract $(70 \mathrm{mg})$. For purification, the crude extract was subjected to silica gel column chromatography and eluted with a gradient of $\mathrm{MeOH} / \mathrm{CH}_{2} \mathrm{Cl}_{2}(9: 1$ to $1: 1)$ to give 40 fractions. The pooled fractions $28-34$ were further purified on a semi-preparative $\mathrm{HPLC}\left(\mathrm{H}_{2} \mathrm{O} / \mathrm{CH}_{3} \mathrm{CN}\right.$ (90:10 to 0:100 in $15 \mathrm{~min}$ ) to yield $\mathbf{2}(1 \mathrm{mg})$.

\section{LC-MS analysis of secondary metabolites}

For the analysis of secondary metabolites via LC-MS, an Agilent 1260 series HPLC equipped with a Multospher 120 RP $18-5 \mu$ column $(250 \times 2 \mathrm{~mm}$, CS-Chromatographie Service $\mathrm{GmbH}$ ) was used. The mobile phase consists of solvent $\mathrm{A}\left(\mathrm{H}_{2} \mathrm{O}\right.$ with $\left.0.1 \% \mathrm{HCOOH}\right)$ and solvent $\mathrm{B}\left(\mathrm{CH}_{3} \mathrm{CN}\right.$ with $0.1 \% \mathrm{HCOOH}$ ) at a flow rate of $0.25 \mathrm{~mL} / \mathrm{min}$. The substances were eluted using a linear gradient from 5 to 100\% B within 40 min. For mass determination, positive ion mode electrospray ionisation (ESI) in a micrOTOF-Q III mass spectrometer with ESI-source (Bruker Daltonics) was used with $5 \mathrm{mM}$ sodium formiate for mass calibration. LC-MS data were evaluated with DataAnalysis 4.2 software (Bruker Daltonik, Bremen, Germany).

\section{NMR analysis}

Isolated compounds were dissolved in DMSO- $\mathrm{d}_{6}$ for structure elucidation via ${ }^{1} \mathrm{H}-\mathrm{NMR}$ analysis. NMR spectra (Figs. S5 and S6) were taken on a JEOL ECA-500 MHz spectrometer (JEOL, Tokyo, Japan) and processed using MestReNova 6.1.0 (Mestrelab). All chemical shifts (Table S4) are referenced to the shift of the solvent signal.

\section{Results}

\section{Construction of $P$. crustosum ku70 (pcr4870) deletion strain using split marker approach}

To improve gene-targeting efficiency in Penicillium crustosum, we aimed to identify and delete the gene coding for the DNA repair protein subunit Ku70. Ku70 as part of the Ku70/ $\mathrm{Ku} 80$ heterodimer is involved in the recognition of DNA double-strand breaks and increases non-homologous DNA end-joining events (Pannunzio et al. 2018). This DNA repair mechanism prohibits homologous recombination events in $P$. crustosum. Deletion of $k u 70$ has been proven to increase the gene-targeting frequency in many fungal species including Penicillium marneffei (Bugeja et al. 2012) and Penicillium digitatum (Gandía et al. 2016).

To find a Ku70 candidate, the putative Ku70 homologue CRL31047.1 from Penicillium camemberti was used for BLAST search, leading to the identification of KAF7525404.1 comprising 653 residues from P. crustosum strain G10 with a sequence identity of $98.6 \%$ on the amino 
acid level. An orthologue Pcr4870 was identified in the genome sequence of $P$. crustosum PRB-2 and differs from KAF7525404.1 at only two residues. Proline at position 43 and threonine at 454 in KAF7525404.1 were replaced by serine and isoleucine in Pcr4870, respectively. Nucleotide sequence comparison revealed differences at four positions. C182, C1463, G1836, and G2071 in the gene coding for KAF7525404.1 were replaced by T182, T1463, A1836, and A2071 in pcr4870 in strain PRB-2, respectively.

Taking into consideration to use the $\Delta p c r 4870$ strain for future gene deletion experiments due to its improved gene targeting, we designed deletion constructs according to the protocol of Maruyama and Kitamoto (2008) to allow multiple gene disruptions by using selection marker recycling. For the deletion of pcr4870, a plasmid was designed containing the $1.5 \mathrm{kbp}$ flanking regions of pcr 4870 and the afpyr $G$ selection marker originated from Aspergillus fumigatus. In addition, a $0.3 \mathrm{kbp}$ fragment of the downstream region is cloned between the upstream sequence and the afpyrG marker to allow subsequent marker recycling as reported by Maruyama and Kitamoto. However, construction of the desired plasmid in both yeast and $E$. coli was unsuccessful. After closely reviewing the cloning strategy described by Maruyama and Kitamoto, it became obvious that inevitable recombination between the $0.3 \mathrm{kbp}$ homologous sequences of the downstream region led to the excision of the afpyrG marker during the cloning procedure. To avoid having the $0.3 \mathrm{kbp}$ homologous regions in one construct, we designed new plasmids using the split marker approach in which each plasmid contains two-thirds of the afpyr $G$ marker, next to the up- or downstream sequences of pcr4870, respectively. The $0.3 \mathrm{kbp}$ fragment of the downstream region is cloned between the upstream sequence and the selection marker to allow subsequent marker recycling by homologous recombination as shown in Fig. 2.

The linearised DNA fragments for disruption of the pcr4870 gene were introduced into the $P$. crustosum $\Delta$ pyrG strain FK15 and selection based on uracil and uridine auxotrophy resulted in $\Delta$ pcr4870::afpyrG strain
JZ02p. Subsequently, selection on plates supplemented with 5-FOA, uracil, and uridine led to the excision of the afpyrG marker by recombination between the $0.3 \mathrm{kbp}$ homologous regions before and after the afpyrG sequence to yield $\Delta$ pcr4870 $\Delta$ afpyrG strain JZ02. Transformants were confirmed by PCR verification as shown in Figs. S1 and $\mathrm{S} 2$.

\section{Inactivation of traB in $P$. crustosum led to the accumulation of viridicatic acid}

As aforementioned, the putative cytochrome $\mathrm{P} 450$ enzyme TraB (Pcr11010) in P. crustosum was speculated to be responsible for the formation of $\mathbf{2}$ in the biosynthesis of 3 (Fig. 1) (Fan et al. 2019). However, this hypothesis was not proven experimentally. We therefore decided to repeat the gene deletion experiment. To maintain the option of further gene deletions in the $\operatorname{traB}$ disruption strain using the same selection marker, we designed split marker constructs for subsequent marker recycling as described above. The $1.5 \mathrm{kbp}$ upstream flanking region of $t \mathrm{ra} B$ and $300 \mathrm{bp}$ from the downstream sequence were amplified from genomic DNA of $P$. crustosum wild-type strain PRB2 , fused with two-thirds of the afpyr $G$ marker and cloned into linearised vector pESC-URA. The second split marker plasmid contained $1.5 \mathrm{kbp}$ of the downstream flanking region and another two-thirds of the afpyr $G$ selection marker. Both DNA fragments for deletion of $t r a B$ were linearised and introduced into the $P$. crustosum pcr4870 disruption strain JZ02, resulting in $\Delta$ pcr4870 $\operatorname{traB}::$ afpyrG strain JZ07. Transformants were selected based on uracil/uridine auxotrophy and additionally verified by PCR (Fig. S3). Cultivation of JZ07 in rice and PDB medium revealed that the inactivation of TraB almost completely abolished the production of $\mathbf{2}$ and $\mathbf{3}$ when compared to the secondary metabolite profile of $\Delta p c r 4870 \Delta$ afpyrG strain JZ02. Instead, clear accumulation of $\mathbf{1}$ could be detected (Fig. 3). Structural elucidation via LC-MS and ${ }^{1} \mathrm{H}-\mathrm{NMR}$
Fig. 2 Strategy for deletion of pcr4870 in P. crustosum using split marker approach followed by selection marker recycling

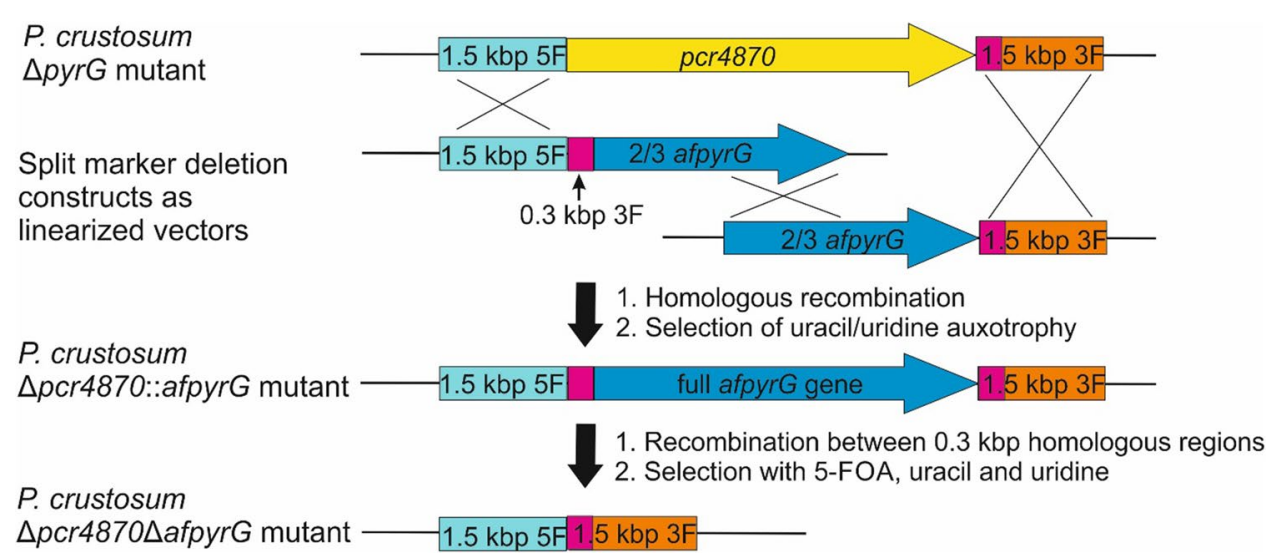

P. crustosum $\Delta$ pyrG mutant

Split marker deletion constructs as linearized vectors 
Fig. 3 LC-MS detection of secondary metabolites from P. crustosum strains. 14-day liquid PDB surface culture of $\Delta$ traB $\Delta$ pcr4870 strain JZ07 (A) and $\Delta$ pcr4870 strain JZ02 (B)

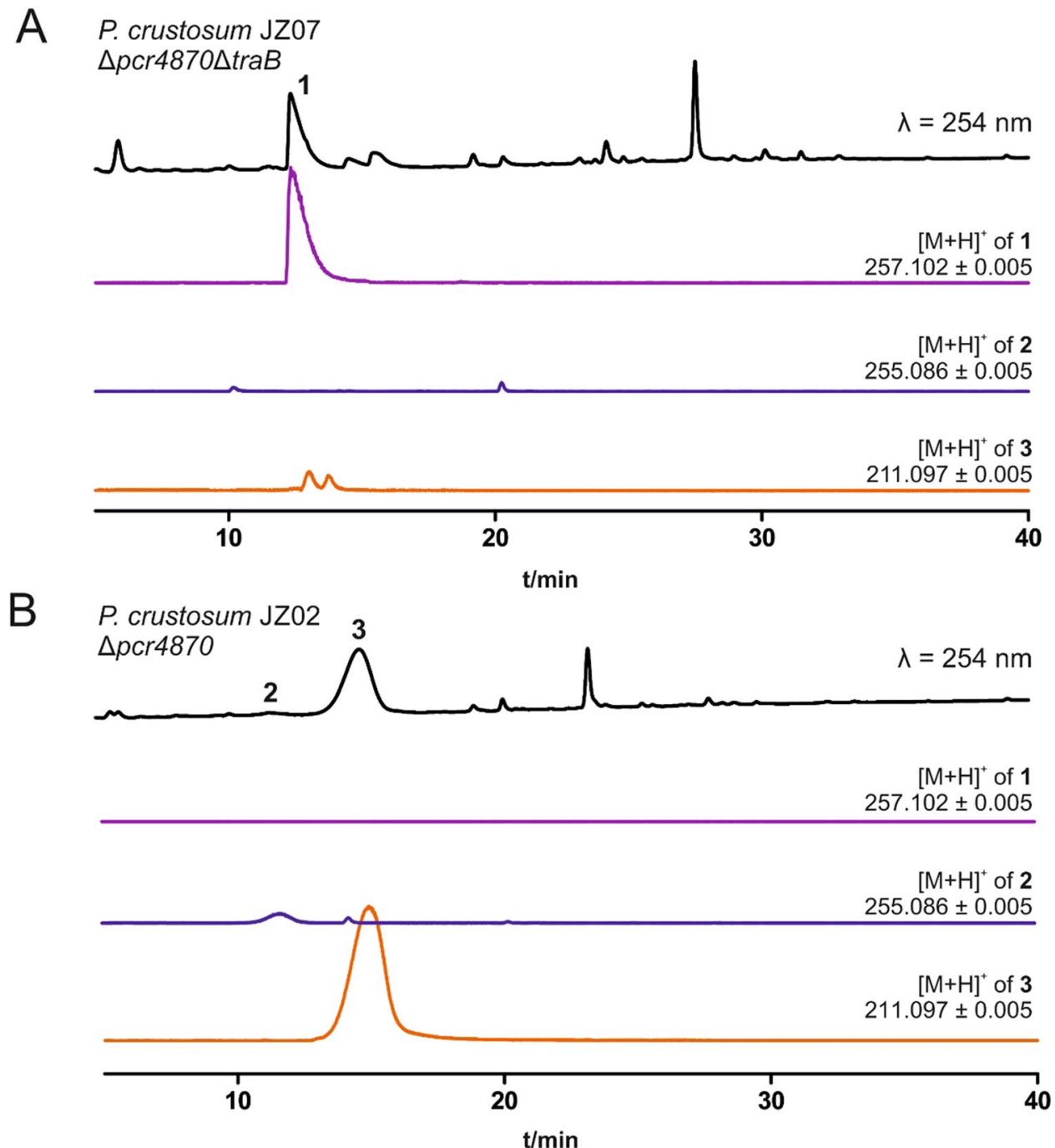

analyses (Fig. S5, Table S4) confirmed 1 to be (5S)-viridicatic acid (Fan et al. 2020).

\section{Heterologous expression of traB in $A$. nidulans and feeding of viridicatic acid led to crustosic acid formation}

Having identified the involvement of TraB in the terrestric acid biosynthesis, we proceeded to investigate the conversion of 1 to $\mathbf{2}$ by heterologous expression of TraB in Aspergillus nidulans LO8030. For this purpose, the whole genomic sequence of $t r a B$ including its native terminator was amplified from $P$. crustosum wild-type strain PRB-2 and cloned into the expression vector pJN017 under the control of the gpdA promoter. The expression construct was then linearised and sitespecifically introduced into the $w A$ locus of $A$. nidulans strain LO8030, resulting in A. nidulans traB expression strain JZ01. Transformants were selected based on riboflavin auxotrophy, and those showing an albino phenotype, indicating successful integration into the $w A$ locus, were additionally verified via PCR (Fig. S4). In parallel, empty vector pJN017 was linearised and introduced into the $w A$ locus of $A$. nidulans to give control strain BK06, which was kindly provided by Bastian Kemmerich from our group.

To analyse the function of TraB, substrate 1 was dissolved in DMSO and fed to the cultures of both overexpression strain JZ01 and control strain BK06 in PDB medium directly after inoculation. After incubation for 7 days, secondary metabolites were extracted with ethyl acetate and analysed via LC-MS. As expected, feeding $\mathbf{1}$ in the $\operatorname{traB}$ overexpression strain led to the formation of $\mathbf{2}$, which was not observed in empty vector control strain BK06 (Fig. 4). For structure confirmation, 2 was isolated from the $t r a B$ overexpression strain JZ01 after feeding with 1 and analysed via LC-MS and ${ }^{1} \mathrm{H}-\mathrm{NMR}$ (Fig. S6, Table S4), proving it unequivocally as $(5 S, 5$ ' $)$ )-crustosic acid (Fan et al. 2019). 
Fig. 4 LC-MS detection of secondary metabolites from $A$. nidulans strains after feeding with 1.7-day liquid PDB surface culture of $\operatorname{traB}$ overexpression strain JZ01 (A) and empty vector control BK06 (B)
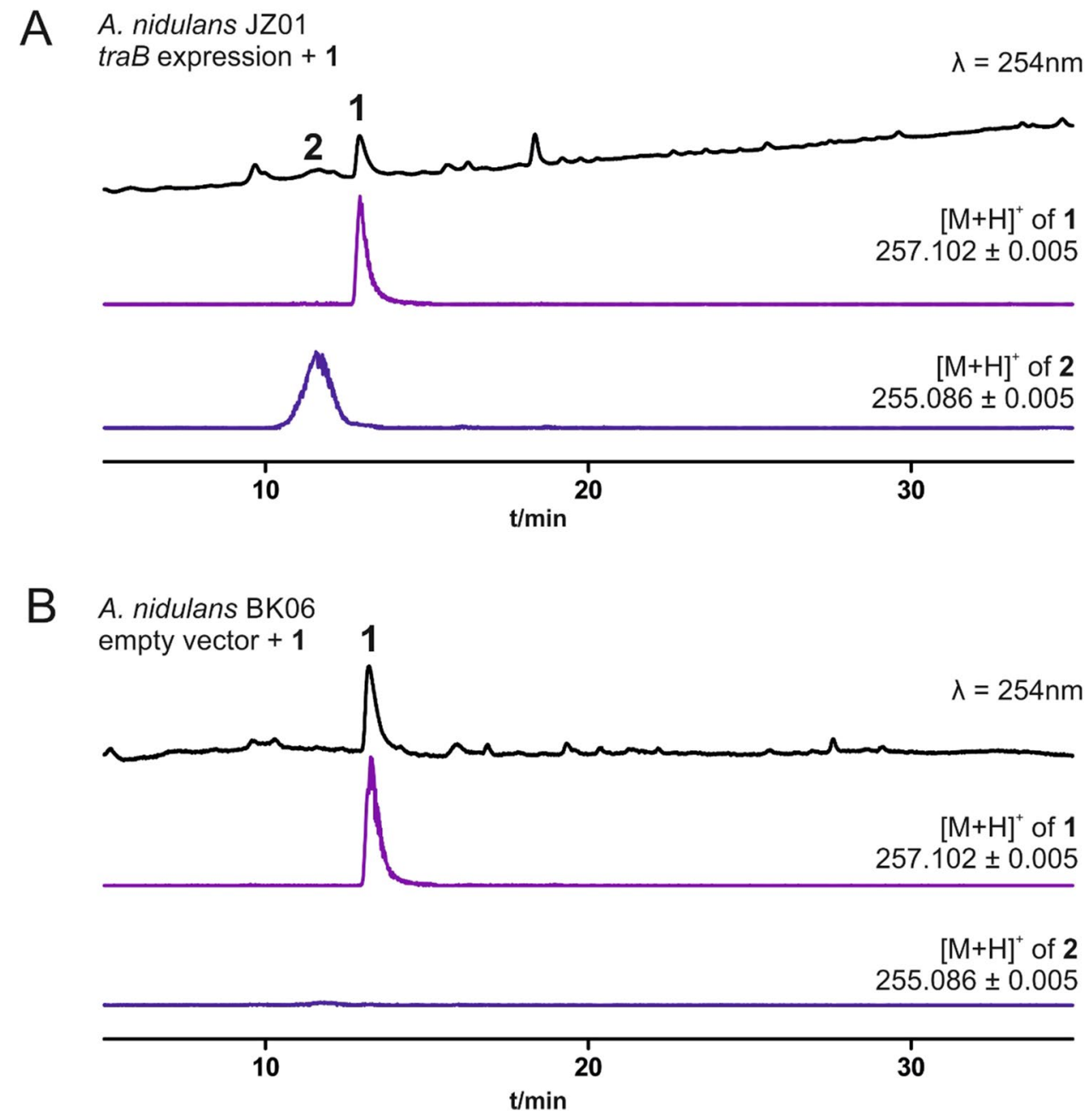

\section{Discussion}

Cytochrome P450 enzymes largely contribute to the high structural diversity of natural products as they catalyse the oxidation of $\mathrm{C}-\mathrm{H}$ groups into more active functional groups (Crešnar and Petric 2011). These P450-mediated functionalisation reactions of unactivated carbon skeletons significantly increase the reactivity and are therefore often essential to provide substrates for tailoring reactions, demonstrating the role of P450s as key enzymes in many biosynthetic pathways (Münch et al. 2021).

In a previous study, our group has demonstrated that the hydroxylation reaction is crucial for the formation of $\mathbf{2}$. By incubating $\mathbf{2}$ in $\mathrm{D}_{2} \mathrm{O}$, it was observed that $\mathbf{2}$ is a closed, anhydrous form of a hydroxylated derivative of $\mathbf{1}$ (Fan et al. 2020). It is very likely that hydroxylated $\mathbf{1}$ can be converted to 2 by spontaneous ring formation. Our main object in this study was the identification of the enzyme responsible for the hydroxylation reaction in the aliphatic side chain of $\mathbf{1}$, the last unknown step in the terrestric acid biosynthesis. As hydroxylation is typically catalysed by
$\mathrm{P} 450$ s, it was speculated that the $\mathrm{P} 450 \mathrm{TraB}$ as a member of the terrestric acid cluster is involved in this reaction.

To facilitate targeted genetic manipulation in P. crustosum, we first tried to delete the gene encoding for DNA repair subunit protein Ku70 using the marker recycling strategy according to the protocol of Maruyama and Kitamoto (2008). However, recombination between the 0.3 kbp homologous regions during the cloning procedure required application of the split marker approach to obtain the desired constructs in two plasmids. Our split marker approach for the construction of deletion plasmids with subsequent marker recycling is applicable for standard cloning strategies with strains like DH5 $\alpha$. In addition, using split marker plasmids for transformation enhances gene targeting due to the requirement of more homologous recombination events in comparison to using a single plasmid. In Penicillium chrysogenum, synergistic effects on gene-targeting efficiency have been reported for applying split marker constructs for the transformation of mutants with a $\Delta k u 70$ or $\Delta l i g D$ background (de Boer et al. 2010). 


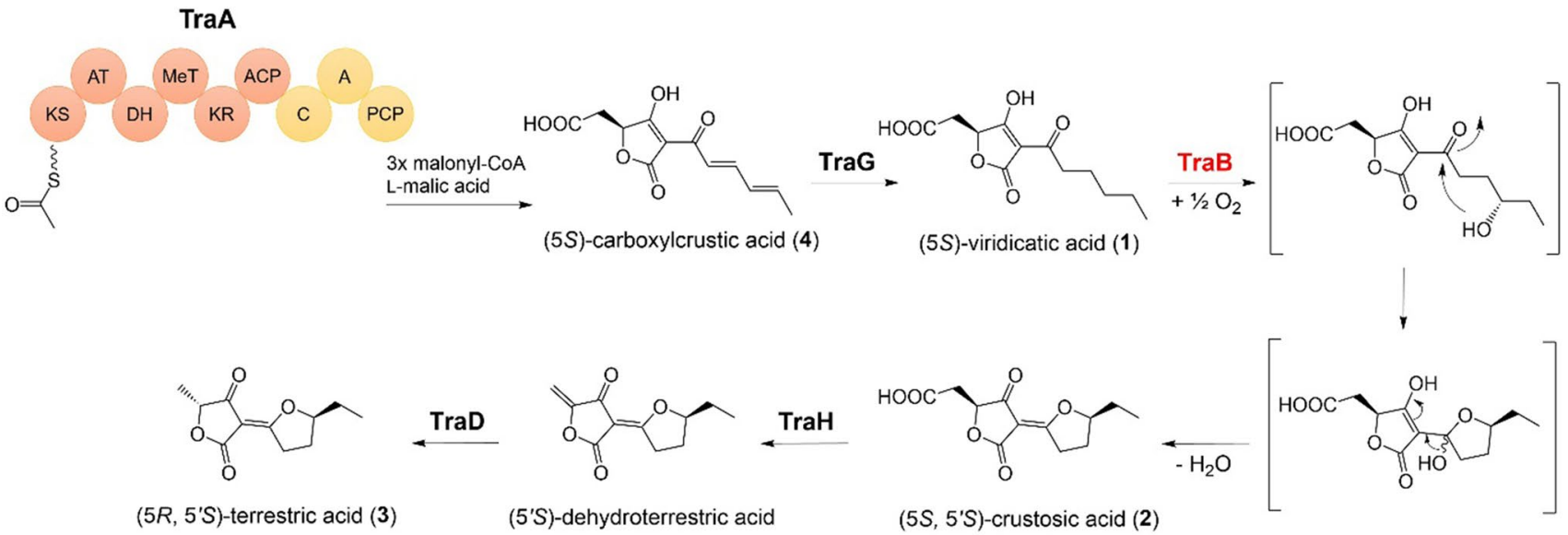

Fig. 5 Biosynthetic pathway of terrestric acid in P. crustosum PRB-2

Our gene deletion experiment in native producer $P$. crustosum proved the role of TraB as the key enzyme for the hydroxylation of $\mathbf{1}$, as accumulation of $\mathbf{1}$ was detected after deletion of $t r a B$. Heterologous expression of $t r a B$ in host organism $A$. nidulans and feeding of the substrate $\mathbf{1}$ led to the formation of $\mathbf{2}$, confirming that TraB alone is responsible for hydroxylation of $\mathbf{1}$. Nucleophilic addition of the hydroxyl group to the carbonyl group at the side chain and subsequent dehydration result in $\mathbf{2}$ (Fig. 5).

Taken together, we optimised the marker recycling strategy by Maruyama and Kitamoto (2008), so that it can be applied for conventional cloning methods. In addition, we improved gene-targeting frequency by deletion of the gene for non-homologous end-joining events. We proved TraB as viridicatic acid hydroxylase by gene deletion in the producer $P$. crustosum and heterologous expression in A. nidulans. With this study, we completely elucidated the reaction steps in the biosynthesis of terrestric acid with their responsible enzymes (Fig. 5).

Supplementary Information The online version contains supplementary material available at https://doi.org/10.1007/s00253-021-11674-4.

Acknowledgements We thank Lena Ludwig-Radtke for taking MS spectra and for the help in the isolation of compounds as well as Stefan Newel for taking NMR spectra. We also thank Bastian Kemmerich for providing A. nidulans control strain BK06.

Author contribution SL is responsible for the project and JZ carried out the experiments. The manuscript was written through contributions of all authors. Both authors have given approval to the final version of the manuscript.

Funding Open Access funding enabled and organized by Projekt DEAL. This project was financially funded in part by the DFG (Li844/11-1 and INST 160/620-1).

\section{Declarations}

Human and animal rights This work does not contain any studies with human participants or animals performed by any of the authors.

Conflict of interest The authors declare no competing interests.

Open Access This article is licensed under a Creative Commons Attribution 4.0 International License, which permits use, sharing, adaptation, distribution and reproduction in any medium or format, as long as you give appropriate credit to the original author(s) and the source, provide a link to the Creative Commons licence, and indicate if changes were made. The images or other third party material in this article are included in the article's Creative Commons licence, unless indicated otherwise in a credit line to the material. If material is not included in the article's Creative Commons licence and your intended use is not permitted by statutory regulation or exceeds the permitted use, you will need to obtain permission directly from the copyright holder. To view a copy of this licence, visit http://creativecommons.org/licenses/by/4.0/.

\section{References}

Atanasov AG, Zotchev SB, Dirsch VM, Supuran CT (2021) Natural products in drug discovery: advances and opportunities. Nat Rev Drug Discov 20:200-216

Bugeja HE, Boyce KJ, Weerasinghe H, Beard S, Jeziorowski A, Pasricha S, Payne M, Schreider L, Andrianopoulos A (2012) Tools for high efficiency genetic manipulation of the human pathogen Penicillium marneffei. Fungal Genet Biol 49:772-778

Chiang YM, Ahuja M, Oakley CE, Entwistle R, Asokan A, Zutz C, Wang CC, Oakley BR (2016) Development of genetic dereplication strains in Aspergillus nidulans results in the discovery of aspercryptin. Angew Chem Int Ed Engl 55:1662-1665

Crešnar B, Petric S (2011) Cytochrome P450 enzymes in the fungal kingdom. Biochim Biophys Acta 1814:29-35

de Boer P, Bastiaans J, Touw H, Kerkman R, Bronkhof J, van den Berg M, Offringa R (2010) Highly efficient gene targeting in 
Penicillium chrysogenum using the bi-partite approach in $\Delta \operatorname{lig} 4$ or $\Delta$ ku70 mutants. Fungal Genet Biol 47:839-846

Fan J, Liao G, Kindinger F, Ludwig-Radtke L, Yin W-B, Li S-M (2019) Peniphenone and penilactone formation in Penicillium crustosum via 1,4-Michael additions of ortho-quinone methide from hydroxyclavatol to $\gamma$-butyrolactones from crustosic acid. J Am Chem Soc 141:4225-4229

Fan J, Liao G, Ludwig-Radtke L, Yin W-B, Li S-M (2020) Formation of terrestric acid in Penicillium crustosum requires redox-assisted decarboxylation and stereoisomerization. Org Lett 22:88-92

Gandía M, Xu S, Font C, Marcos JF (2016) Disruption of ku70 involved in non-homologous end-joining facilitates homologous recombination but increases temperature sensitivity in the phytopathogenic fungus Penicillium digitatum. Fungal Biol 120:317-323

Harken L, Li S-M (2021) Modifications of diketopiperazines assembled by cyclodipeptide synthases with cytochrome P450 enzymes. Appl Microbiol Biotechnol 105:2277-2285

Katz L, Baltz RH (2016) Natural product discovery: past, present, and future. J Ind Microbiol Biotechn 43:155-176

Keller NP (2019) Fungal secondary metabolism: regulation, function and drug discovery. Nat Rev Microbiol 17:167-180

Kindinger F, Nies J, Becker A, Zhu T, Li S-M (2019) Genomic locus of a Penicillium crustosum pigment as an integration site for secondary metabolite gene expression. ACS Chem Biol 14:1227-1234

Maruyama J, Kitamoto K (2008) Multiple gene disruptions by marker recycling with highly efficient gene-targeting background $(\Delta l i g D)$ in Aspergillus oryzae. Biotechnol Lett 30:1811-1817
McIntosh JA, Farwell CC, Arnold FH (2014) Expanding P450 catalytic reaction space through evolution and engineering. Current Opin Chem Biol 19:126-134

Münch J, Püllmann P, Zhang W, Weissenborn MJ (2021) Enzymatic hydroxylations of $\mathrm{sp}^{3}$-carbons. ACS Catal 11:9168-9203

Pannunzio NR, Watanabe G, Lieber MR (2018) Nonhomologous DNA end-joining for repair of DNA double-strand breaks. J Biol Chem 293:10512-10523

Podust LM, Sherman DH (2012) Diversity of P450 enzymes in the biosynthesis of natural products. Nat Prod Rep 29:1251-1266

Shabana S, Lakshmi KR, Satya AK (2021) An updated review of secondary metabolites from marine fungi. Mini Rev Med Chem 21:602-642

Wu G, Ma H, Zhu T, Li J, Gu Q, Li D (2012) Penilactones A and B, two novel polyketides from Antarctic deep-sea derived fungus Penicillium crustosum PRB-2. Tetrahedron 68:9745-9749

Yu G, Sun Z, Peng J, Zhu M, Che Q, Zhang G, Zhu T, Gu Q, Li D (2019) Secondary metabolites produced by combined culture of Penicillium crustosum and a Xylaria sp. J Nat Prod 82:2013-2017

Zhang X, Guo J, Cheng F, Li S (2021) Cytochrome P450 enzymes in fungal natural product biosynthesis. Nat Prod Rep 38:1072-1099

Publisher's note Springer Nature remains neutral with regard to jurisdictional claims in published maps and institutional affiliations. 